\title{
Correction to: Detection of Fusobacterium nucleatum in stool and colonic tissues from Norwegian colorectal cancer patients
}

\author{
Hege Smith Tunsjø $\varnothing^{1,2} \cdot$ Gro Gundersen $^{3} \cdot$ Fredrik Rangnes $^{3} \cdot$ John Christopher Noone ${ }^{2,4,5} \cdot$ Alexander Endres $^{6}$. \\ Vahid Bemanian ${ }^{3}$
}

Published online: 28 November 2019

(C) Springer-Verlag GmbH Germany, part of Springer Nature 2019

Correction to: European Journal of Clinical Microbiology \& Infectious Diseases (2019) 38:1367-1376.

https://oi.org/10.1007/s10096-019-03562-7

Two affiliations of author John Christopher Noone were not included in the original article and have been added here. Please see below.

Also, Acknowledgements of the originally published article is not complete. Please see the corrected section below.

The online version of the original article can be found at https://oi.org/ 10.1007/s10096-019-03562-7

Hege Smith Tunsjø

hetu@ oslomet.no

Department of Life Sciences and Health, Oslo Metropolitan

University, Oslo, Norway

2 Department of Microbiology and Infection Control, Akershus University Hospital, Lørenskog, Norway

3 Department of Multidisciplinary Laboratory Science and Medical Biochemistry, Genetic Unit, Akershus University Hospital, Lørenskog, Norway

4 Faculty of Medicine, University of Oslo, Oslo, Norway

5 Department of Clinical Molecular Biology (EpiGen), Division of Medicine, Akershus University Hospital and University of Oslo, Oslo, Norway

6 Department of Gastroenterology, Akershus University Hospital, Lørenskog, Norway

\section{Acknowledgements}

Students at Oslo Metropolitan University (Mia Sandgren et al., Sammy Ramstock et al., and Silje Mathiassen et al.) have contributed to parts of the contents in this manuscript. We thank the Pathology Department at Akershus University Hospital (Ahus) for characterization of tumors, and Tone Møller Tannes and Aina EF Moen at the Department of Clinical Molecular Biology (EpiGen), Ahus, for DNA extraction of biopsies. We also express our gratitude to biomedical laboratory scientist Eva Smedsrud, Department of Multidisciplinary Laboratory Science and Medical Biochemistry, Genetic Unit, for all the help with patient inclusion and sample processing. 